\title{
Second-order wave maker theory using force-feedback control. Part II. An experimental verification of regular wave generation.
}

\author{
Johannes Spinneken and Chris Swan \\ Imperial College London, Department of Civil and Environmental Engineering \\ London SW7 2AZ
}

2009

\begin{abstract}
This paper provides an experimental verification of the new wave maker theory outlined by Spinneken \& Swan (2008a). This theory concerns the generation of regular waves by a flap-type wave maker using force-feedback control, providing the first quantitative evidence of the inherent advantages of this latter approach. When the wave maker is controlled by a first-order force command signal, comparisons between the theory and experimental observations confirm two key points: (i) The first-order behaviour is crucial for the absorption characteristics of the machine. (ii) The second-order behaviour leads to a spurious, or unwanted, freely propagating second harmonic that is substantially smaller in amplitude when compared to an identical wave paddle operating with first-order position control. Both aspects of this work, effective absorption and reduced second-order spurious wave generation, are investigated over a broad range of wave frequencies and shown to be widely applicable. Furthermore, the theory also provides a force command signal correct to second order. This is introduced in a separate set of experiments and shown to provide further improvement in the quality of the wave generation.
\end{abstract}

Keywords: Laboratory wave generation, Second-order wave maker theory, Force-feedback control, Impedance matching.

\section{Introduction}

In an earlier paper, hereafter referred to as part I, Spinneken \& Swan (2008a) developed a second-order wave maker theory incorporating force-feedback control. The motivation for this work was the need for an improved understanding of wave generation, driven by a desire to accurately control the wave forms produced within a laboratory environment. Indeed, recent research has demonstrated that the precise control of a generated wave field is fundamental to the analysis of:

i. The nonlinear wave-wave interactions associated with the evolution of the largest or most extreme wave events (Baldock et al. (1996), Johannessen \& Swan (2001),(2003)).

ii. The nonlinear wave-structure or wave-vessel interactions associated with the imposition of the largest loads, the loss of an air-gap, or the occurrence of wave impacts (Swan et al. (1997), Sheikh \& Swan $(2005))$.

Given the motivation of this work, it is clear that several aspects of the theory developed in part I require experimental validation. First, the active absorption of wave components by a wave maker controlled by force-feedback is dependent upon first-order impedance matching. Although the underlying theory has been known for several decades and has been adopted in many applications, including wave generation, it has not been thoroughly discussed in the context of actively absorbing wave machines. The success of this approach is addressed in $\S 3$, with comparisons between the theory and the absorption characteristics of a flap-type wave maker.

Second, the spurious second-order wave content produced when a force controlled machine is driven with a 
first-order command signal requires investigation. The theory developed in part I suggests that first-order force control can greatly enhance the wave quality when compared to first-order position control. This proposition is carefully examined in $\S 4$, with direct comparisons between the different control strategies.

Third, the theory developed in part I also provides a second-order correction to the command signal. The success of this approach and the improvement it provides over a broad range of wave conditions is examined in $\S 5$. The paper concludes in $\S 6$ with an overview of the experimental validation and a discussion of future work.

\section{Experimental Investigation}

The experiments were undertaken in a narrow wave flume located in the hydrodynamics laboratory in the Department of Civil and Environmental Engineering at Imperial College London. The wave making facilities at Imperial College London were manufactured and commissioned by Edinburgh Designs Ltd. The control system and methodology driving the wave maker used in the present study are identical to those widely installed by Edinburgh Designs. As such, the results will be directly relevant to the operation of many wave machines and provide guidance as to the effective operation of others.

The wave flume employed in the present investigation is $0.3 \mathrm{~m}$ wide, $27 \mathrm{~m}$ long and equipped with wave boards on either end. Both paddles are bottom hinged and mechanically identical. The water depth was maintained constant at $h=0.7 \mathrm{~m}$ throughout the wave flume. A precision force transducer and a high resolution motor shaft encoder were used to measure the physical quantities of wave board torque and angular position. A flexible digital control system allowed for an uncomplicated and fast change of the systems control program. This reads the digitized signals for torque and position and can process them in any required fashion. As a result, the paddle can be operated in position-control or force-control mode. Furthermore, digital absorption filters were implemented and could be rapidly altered.

The test cases chosen for the experimental verification cover a fairly broad range of frequencies. In shallow water conditions, it is well known that Stokes theory over predicts the magnitude of the second-order components. Schäffer (1996) introduced the nonlinearity parameter $S$; a value of $S=1$ corresponding to the 'limiting' case where second-order Stokes theory predicts secondary peaks in the trough of the primary wave. As a result, for $S>1$ the theory fails to predict the correct second-order content. With the force-feedback model outlined in part I utilizing the wave field solution derived by Schäffer (1996), the nonlinearity factor $S$ will also be applied herein. For a regular wave with angular frequency $\omega=2 \pi f$, wavenumber $k$ and wave height $H$, the nonlinearity factor $S$ is given by

$$
S=2 H\left|\frac{\omega H^{+}}{2 g^{2} k \tanh (2 k h)-4 g \omega^{2}}-\frac{1}{4 g}\left(\frac{g^{2} k^{2}}{\omega^{2}}-3 \omega^{2}\right)\right|
$$

\begin{tabular}{ccccc}
\hline \hline $\begin{array}{c}\text { Wave } \\
\text { frequency, } \\
f[H z]\end{array}$ & $\begin{array}{c}\text { Nondimensional } \\
\text { water depth, } \\
h / L_{0}\end{array}$ & $\begin{array}{c}\text { Wave } \\
\text { height, } \\
H[\mathrm{~mm}]\end{array}$ & $\begin{array}{c}\text { Wave } \\
\text { steepness, } \\
H k / 2\end{array}$ & $\begin{array}{c}\text { Wave } \\
\text { nonlinearity, } \\
S\end{array}$ \\
\hline $32 / 64$ & 0.11 & 148 & 0.10 & 0.60 \\
$34 / 64$ & 0.13 & 150 & 0.11 & 0.57 \\
$36 / 64$ & 0.14 & 164 & 0.13 & 0.59 \\
$38 / 64$ & 0.16 & 176 & 0.15 & 0.60 \\
$40 / 64$ & 0.18 & 174 & 0.16 & 0.57 \\
$42 / 64$ & 0.19 & 162 & 0.16 & 0.52 \\
$44 / 64$ & 0.21 & 150 & 0.16 & 0.47 \\
$46 / 64$ & 0.23 & 142 & 0.16 & 0.45 \\
$48 / 64$ & 0.25 & 132 & 0.16 & 0.42 \\
$50 / 64$ & 0.27 & 124 & 0.16 & 0.40 \\
$52 / 64$ & 0.30 & 116 & 0.16 & 0.38 \\
$54 / 64$ & 0.32 & 108 & 0.16 & 0.36 \\
$56 / 64$ & 0.34 & 102 & 0.16 & 0.35 \\
$58 / 64$ & 0.37 & 96 & 0.16 & 0.34 \\
$60 / 64$ & 0.39 & 92 & 0.16 & 0.34 \\
$62 / 64$ & 0.42 & 88 & 0.17 & 0.35 \\
$64 / 64$ & 0.45 & 82 & 0.17 & 0.34 \\
\hline
\end{tabular}

Table 1: Laboratory test cases 
where $H^{+}$is defined as

$$
H^{+}=2 \omega\left(\omega^{2}-\frac{g^{2} k^{2}}{\omega^{2}}\right)+\omega^{3}-\frac{g^{2} k^{2}}{\omega}
$$

and $g$ denotes acceleration due to gravity.

For the lowest wave frequencies, the steepness is limited by the maximum available stroke of the wave machine. When driven in force-control mode, the maximum wave-generating angle has to be further limited to allow for the additional superimposed motion necessary for the absorption of reflected waves. Overall, a maximum steepness of $H k / 2=0.1$ was adopted for the longest wave having a period of $T=2.0 \mathrm{~s}$. This wave condition lies well within the applicability region of the theory $(S=0.60)$.

Full details of the regular wave cases used in the laboratory study are given in table 1 . For each case the wave frequency $f$, the normalised water depth $h / L_{0}$, the dimensionless steepness $H k / 2$ and the nonlinearity parameter $S$ are shown; where $L_{0}=2 \pi g / \omega^{2}$ is the deep water wave length.

\section{First-order control and wave absorption}

With position-controlled wave machines, the first-order behaviour is well understood; the amplitude of the generated progressive wave being readily evaluated from the position command signal. In contrast, forcecontrolled wave machines require an empirically determined transfer function; details of which are discussed by Masterton \& Swan (2008). Whilst an empirical transfer function may remain the preferred approach for the calibration of a wave flume and, in particular, a 3-D wave basin, in the short term, the model developed in part I provides a first theoretical transfer function linking the demand signal and the first-order surface elevation for force-controlled wave machines. Although this demand signal has the dimension of a torque it actually represents the input signal appropriate to the summation of the hydrodynamic feedback torque and the filtered velocity. As a result, it must not be confused with the actual torque acting on the wave machine. This latter summation may appear dimensionally inconsistent. However, the measured velocity signal passes through the impedance matching absorption filter, $Z_{f}(\omega)$. The impedance $Z_{f}(\omega)$ has the dimension of torque divided by angular velocity (Falnes 2002), and hence the SI unit of $N \mathrm{~m} /(\mathrm{rad} / \mathrm{s})$. Consequently, the filtered velocity has the dimension of torque with a unit of $\mathrm{Nm}$.

Figure 1 compares the theoretical first-order transfer function with experimental data relating to a bottom hinged wave machine. The agreement between the theoretical model and the experimental data is excellent across the broad range of wave cases investigated. On the basis of these results it is clear that, at the very least, the theoretical transfer function provides a very good input for a first iteration of a wave maker calibration.

Part I also derived a first-order impedance matching approach to implement active absorption with flap-type wave boards. Full absorption is achieved if the absorption filter provides a complex conjugate impedance, or negative reactance, to the combined wave-board and wave-field impedance. For the experimental validation a network of digital filters was designed and implemented in the machine's controller. With the filter and the wave-board-water impedance known, the theoretical power reflection coefficient, $\gamma_{r}$, can be calculated; refer to part I for further details. Figure 2 compares the theory with the experimental observations; the data presented in terms of $\gamma_{r}$ vs normalised water depth, or $h / L_{0}$. Overall, the agreement between the theory and the experiment is generally good but becomes less satisfactory for higher frequencies.

In the experimental study the reflection coefficient was calculated using Method I proposed by Isaacson (1991); the wave gauge array consisting of 8 gauges with individual gauges spaced at a fixed distance in all test cases. For the data points shown in figure 2 the dimensionless probe spacing angle varied between $20^{\circ}$ and $110^{\circ}$. On the basis of Isaacson's (1991) figure 4, this relates to analysis errors of approximately $20 \%$. Within figure 2, the larger deviations occurring towards the higher frequencies may be due to an inaccurate estimate of the paddle's inertia since this becomes dominant, or at least progressively more important, in this region. Another key issue neglected in the theoretical model, is the impact of a finite loop gain in the controller. The gain roll off at higher frequencies may affect the machine's response and lead to a non-ideal absorption characteristic. Nevertheless, in spite of these difficulties, the data presented in figure 2 is in generally good agreement with the theory.

In a double ended wave flume the success of active wave absorption based on force-feedback, with simultaneous wave generation, can be demonstrated by the generation of a standing wave of constant shape. The water 


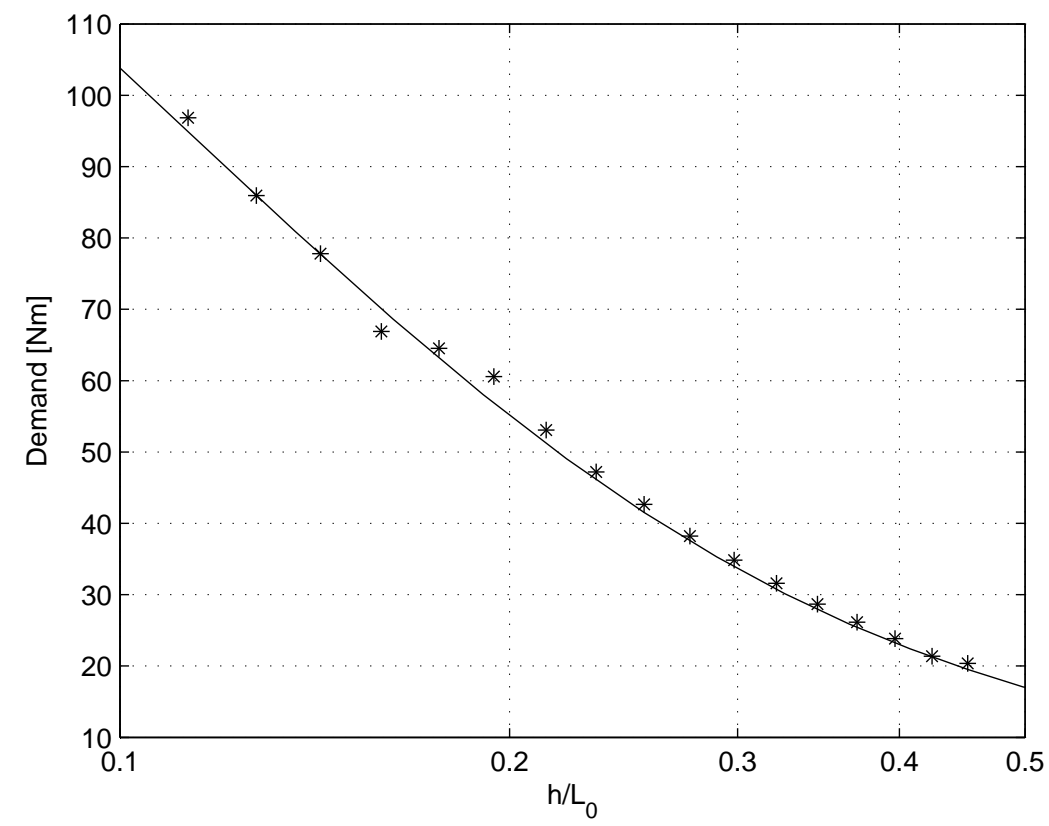

Figure 1: Demand signal at first order to generate a wave of steepness $H k / 2=0.1$ for a bottom hinged wave machine; — Force-control theory; * Experimental transfer function

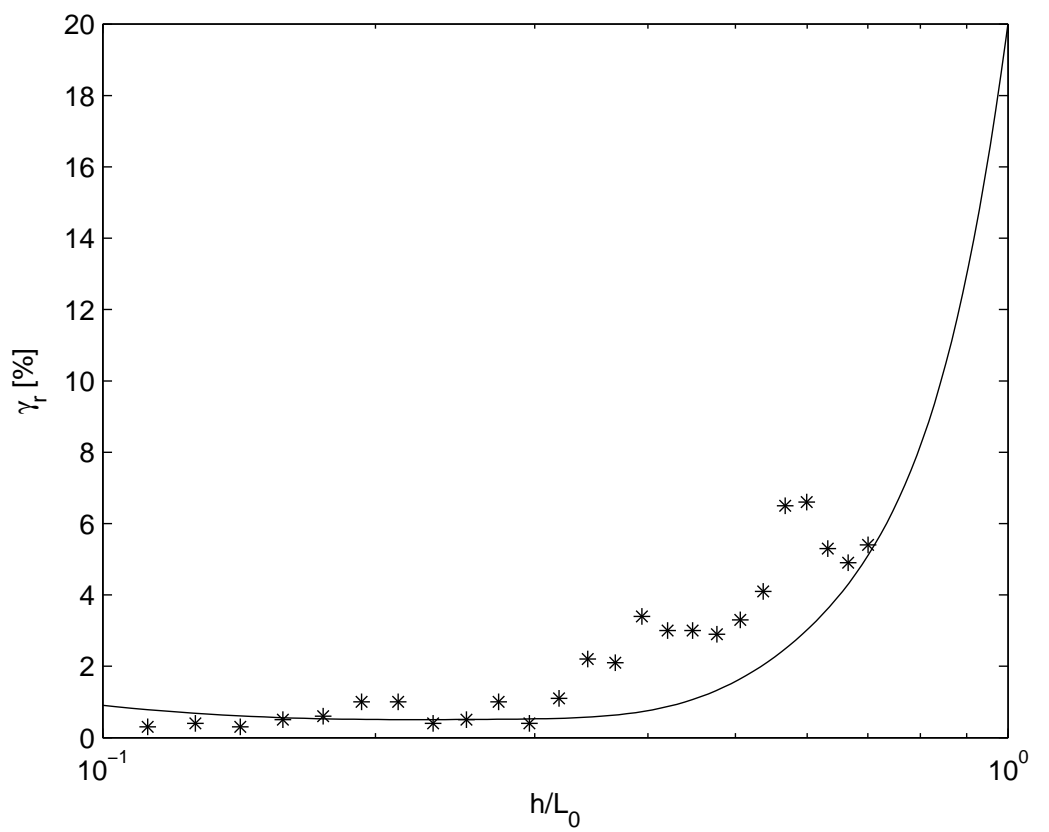

Figure 2: Reflection coefficient — Theoretical power absorption coefficient $\gamma_{r}$ in percent; $*$ Experimental data 

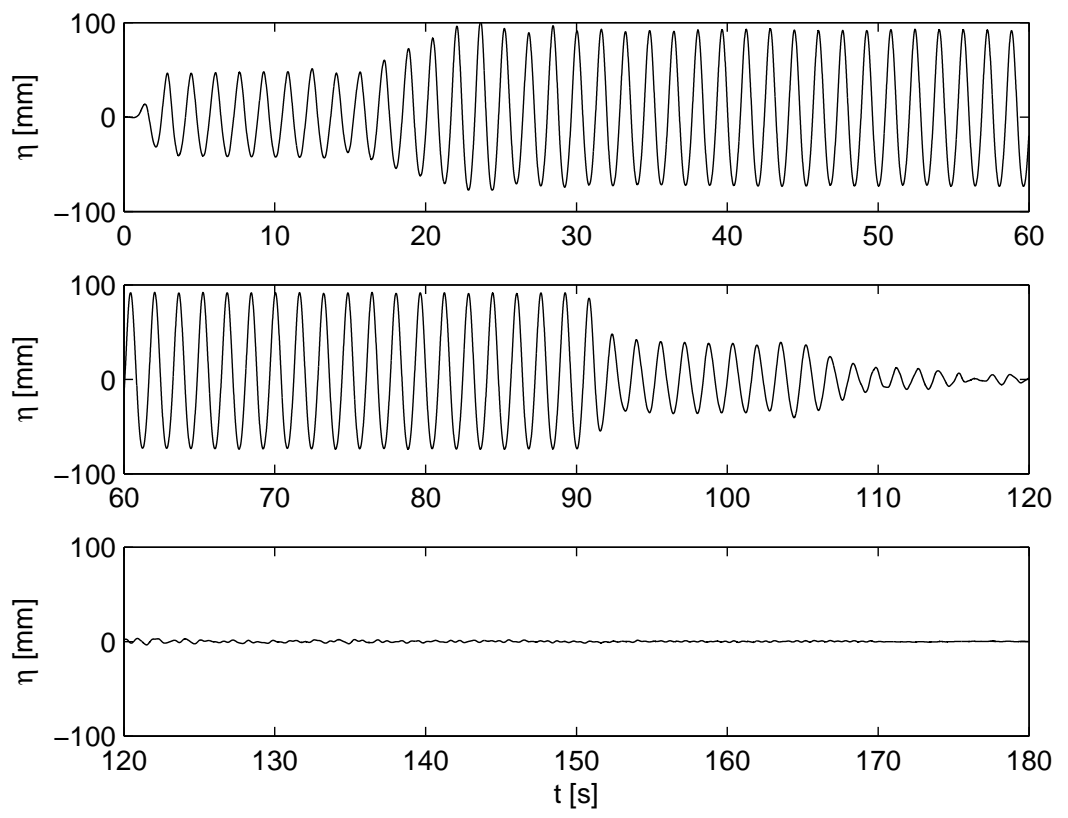

Figure 3: Water surface elevation $\eta(t)$ corresponding to two waves, each with $f=40 / 64 H z$ and steepness $H k / 2=0.08$, travelling in opposite directions. Wave generation commenced at $t=0 \mathrm{~s}$ and stopped at $t=90 \mathrm{~s}$; the wave gauge being located at the first anti-node adjacent to one wave maker. Note: Although the figure is presented as three subplots, they are sequential defining one continuous time-history, $\eta(t)$.

surface profile, $\eta(t)$, recorded by a single wave gauge placed at the first anti-node adjacent to one wave maker is shown in figure 3 . In this example both wave makers commence wave generation simultaneously at $t=0 s$. The generated waves are of identical frequency and amplitude; the resulting interference pattern creating a standing wave. With the wave gauge located much closer to one wave paddle than the other, the entire data record can be sub-divided into the following intervals. During the first time interval $(0 s \leq t \leq 15 s)$ the wave probe records the wave generated by the adjacent wave maker only; a regular wave train being quickly established and maintaining a constant shape and wave height. Subsequently, the wave generated at the opposite end arrives and the standing wave profile progressively develops, becoming fully developed by $t \approx 25 \mathrm{~s}$. In the time interval $25 \mathrm{~s} \leq t \leq 90 \mathrm{~s}$ both wave makers must absorb and generate concurrently in order to maintain the standing wave profile. The resulting surface profile within this interval (figure 3 ) is almost perfectly constant, the standard deviation in the wave height being of order $\sigma=0.17 \mathrm{~mm}$. This indicates that the simultaneous generation and absorption using force-feedback control is both practical and effective. At time $t=90 \mathrm{~s}$ the wave generation was stopped and both wave makers are absorbing only; the surface profile recorded in the interval $90 s \leq t \leq 105 s$ is the wave approaching from the opposite end with almost no reflection occurring from the adjacent wave maker. Almost all of the wave energy in the flume is absorbed by $t=180 \mathrm{~s}$.

\section{Errors arising from a first-order command signal}

In addition to operating as excellent absorbers, force-feedback (or impedance matching) wave machines appear to introduce very little spurious harmonic content when driven with a first-order command signal. For the first time, the model developed in part I explains this apparent advantage but there is a complete absence of laboratory data investigating this effect. To correct this, the spurious wave content was measured for all of the test cases noted in table 1. In each case the wave maker was run in both first-order position control and first-order force control. The resulting data is presented in figure 4: the spurious second-order wave amplitude, $A^{(22)}$, being normalised with respect to the second-order Stokes amplitude, $A^{(21)}$, so that 


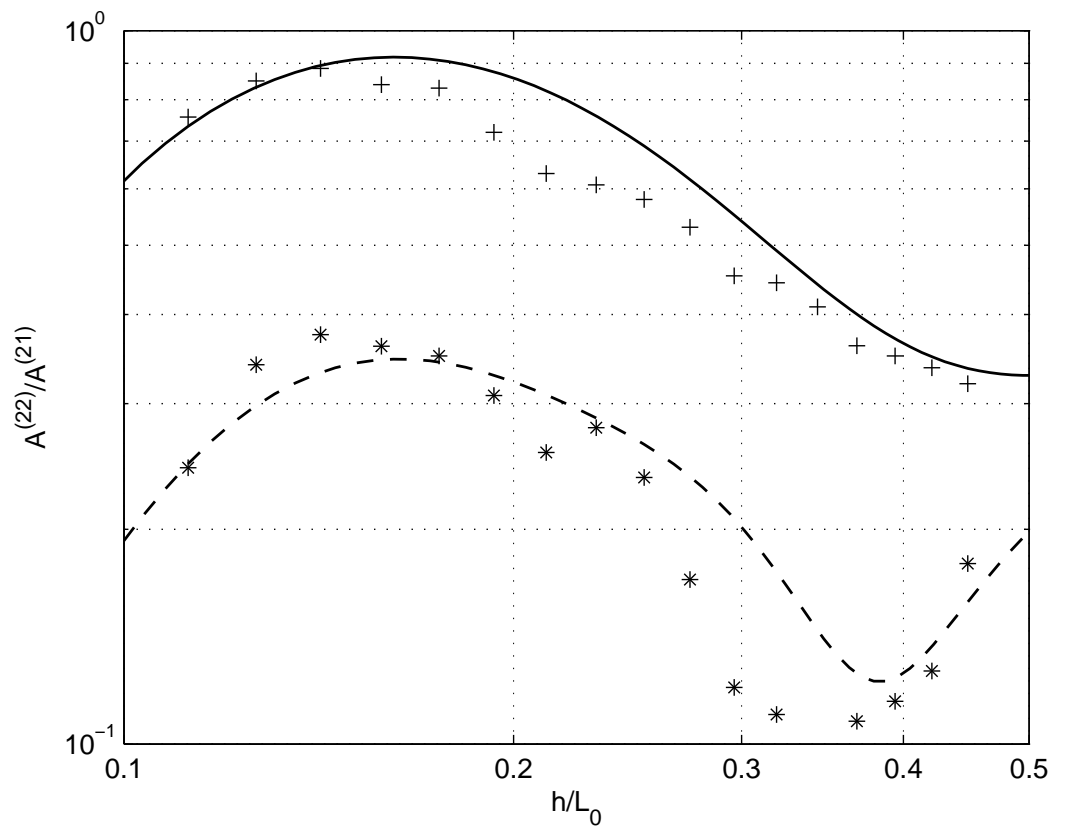

Figure 4: Dimensionless, second-order, spurious wave content for a bottom-hinged wave maker — Positioncontrol theory; + First-order position-control experiment; - - Force-control theory; $*$ First-order forcecontrol experiment

the form of the presentation is independent of wave steepness.

In both control modes (position- and force-control) the agreement between the theoretical results and the experimental data is good across a broad range of frequencies. The observed deviation at the higher frequencies may have arisen for several reasons. First, the impact of the finite loop gain may be partially responsible. Second, when generating very short regular waves a small spurious cross-tank resonance was observed introducing additional measurement errors. Third, given the moderate steepness of the waves, the amplitude of the high frequency waves is relatively small. This, in turn, means that the absolute amplitude of the spurious second-order waves is extremely small (within the sub millimeter range) and this has obvious implications for the relative errors associated with the data acquisition.

Nevertheless, despite these difficulties, the experimental data clearly demonstrates that the operation of a flap-type wave machine with a force-control signal yields a significantly smaller second-order spurious wave content when compared to an identical paddle operating in position-control mode.

However, it must be stressed that for most test cases the relative magnitude of the spurious wave is small. Indeed, the largest values are measured for shallow water waves where the second-order Stokes content is relatively large. Figure 5 concerns the longest wave case investigated in the present study and contrasts first-order position control (a) with first-order force control (b). In both modes the measured water surface profile is compared to second-order Stokes theory. Comparison between these cases demonstrates the much improved wave quality achieved using first-order force control. This is further established in figure 6 which provides a detailed view of an adjacent wave crest and wave trough in both modes of operation; the location of these points being noted as (1) to (4) on figure 5 . With the data sampled at $128 \mathrm{~Hz}$, individual data points are best shown by taking every third measured point. Absolutely no further filtering has been applied to the data in these examples, or any others presented within this paper. In comparing the plots given on figure 6 , the errors associated with the description of the wave trough are much reduced when the wave generation is based on the force controlled mode.

Figure 7 shows the surface profile for a much shorter wave $(f=0.875 \mathrm{~Hz})$ for which the wave steepness has been increased to $H k / 2=0.23$, highlighting the discrepancies in the wave shape arising at second order and above. Figure $7(\mathrm{a})$ shows the water surface profile arising from first-order position control, while figure 7(b) 

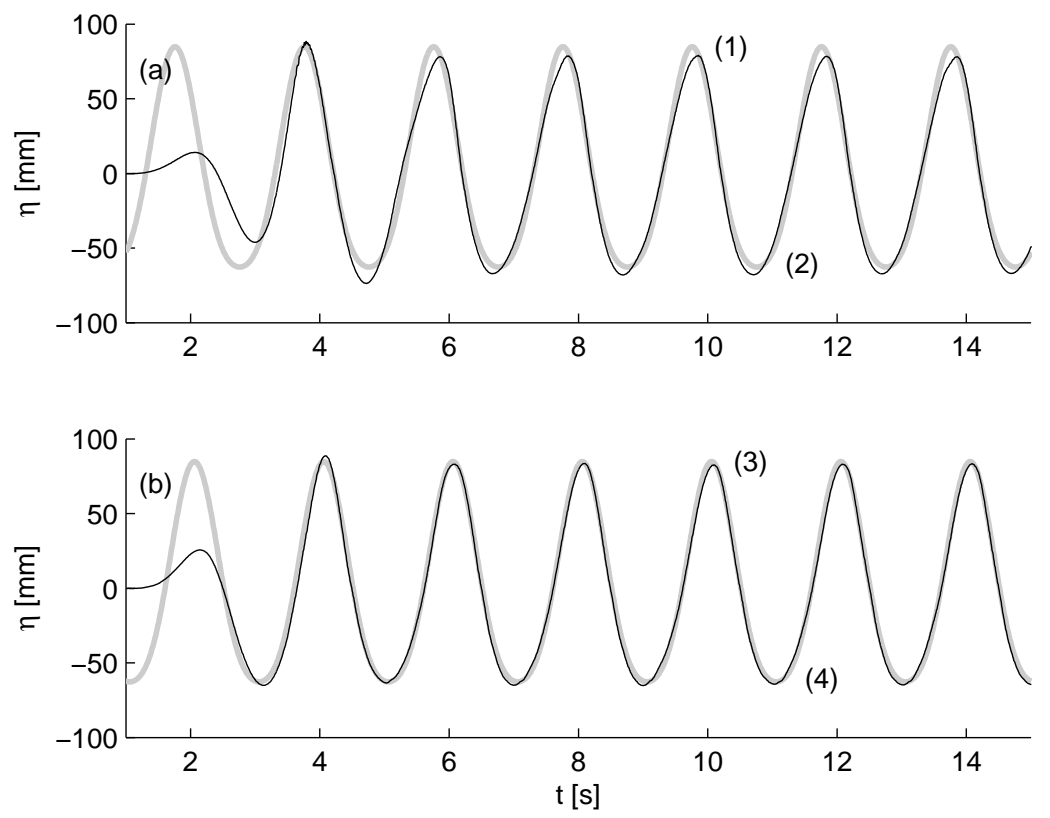

Figure 5: Surface elevation for wave case $f=32 / 64 H z, H k / 2=0.10$; (a) First-order position control, (b) First-order force control; — Experiment, - Second-order Stokes solution; Note: the numbers (1) to (4) refer to locations that are considered in detail in figure 6
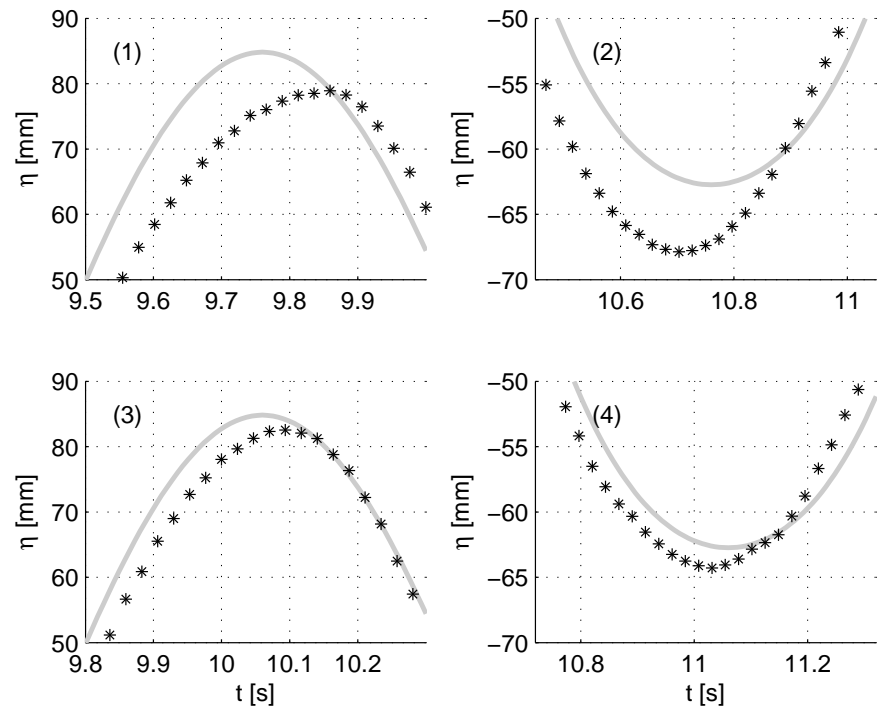

Figure 6: Close-up of the water surface elevation at locations (1) to (4) for wave case $f=32 / 64 \mathrm{~Hz}$, $H k / 2=0.10$ as labelled in figure $5 ; *$ Experiment, $\_$Second-order Stokes solution 

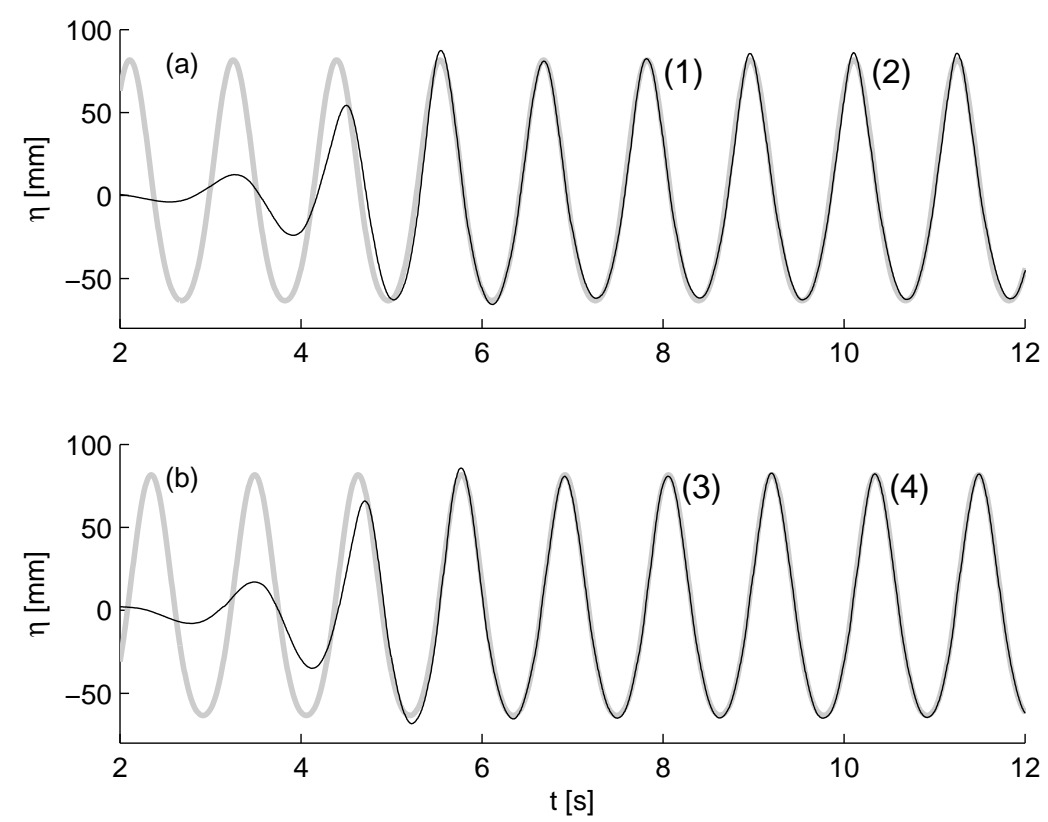

Figure 7: Surface elevation for wave case $f=56 / 64 H z, H k / 2=0.23$; (a) First-order position control, (b) First-order force control; — Experiment, — Second-order Stokes solution; Note: the numbers (1) to (4) refer to locations that are considered in detail in figure 8

presents the corresponding profile produced by first-order force control; in both cases two wave crests are highlighted, numbered (1)-(4), and these are further considered in figure 8. The wave profile generated using first-order position control (figure 7a) is initially in very close agreement with a second-order Stokes' solution; evidence of this is being provided at location (1) on figure 8. However, this agreement is misleading because, given the frequency of the wave involved, any spurious second-harmonic wave evolving in the vicinity of the paddle will have had insufficient time to propagate to the measuring section some $2.5 \mathrm{~m}$ downstream of the paddle. The significance of this effect becomes clear in subsequent wave cycles. For example at location (2) on figure 7(a), the measured data shows marked departure from the Stokes theory, confirming the presence of significant spurious second-order wave components. In contrast, the wave profile generated by first-order force control is in near-perfect agreement with the Stokes solution, showing no evidence of the delayed arrival of unwanted or spurious wave components. Evidence of this is provided by the crest elevation at locations (3) and (4) on figure 8; the contrast between locations (2) and (4) being the key issue. 

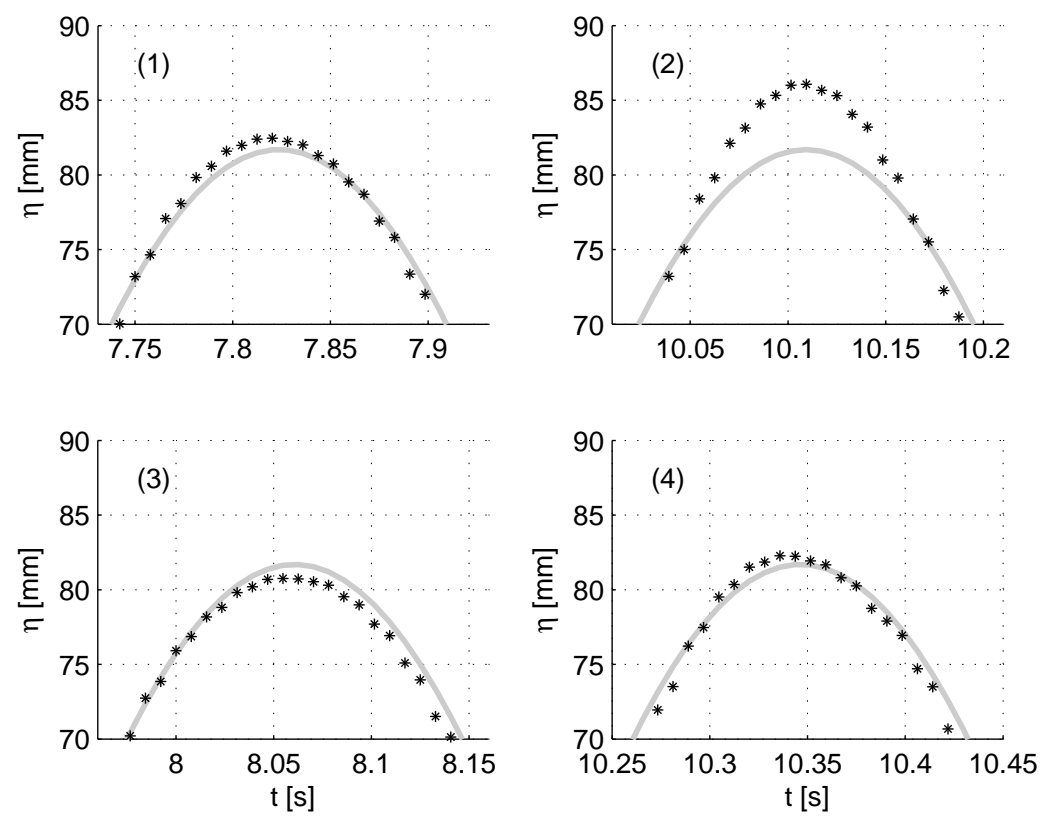

Figure 8: Close-up of the water surface elevation at locations (1) to (4) for wave case $f=56 / 64 H z$, $H k / 2=0.23$ as labelled in figure $7 ; *$ Experiment, $=$ Second-order Stokes solution

\section{Second-order control}

Comparison between the part I theory and the present experiments have shown that using a first-order force control signal introduces very little spurious wave content over a broad frequency range. However, in the shallow water wave cases, where the second-order Stokes contributions is more pronounced, the wave profile remains less than ideal; see, for example, figure 5(b) or more specifically location (4) on figure 6 . In order to correct for the remaining spurious wave, the part I theory provides an expression for the second-order control signal. The applicability of this correction term is considered in this section.

Figure 9 illustrates the benefits of second-order force control. Figure 9(a) concerns the second-order spurious wave amplitude, $A^{(22)}$, non-dimensionalised with respect to the second-order Stokes amplitude, $A^{(21)}$. For reference purposes the results of the first-order command signal are reproduced and it is clear from these comparisons that the second-order command signal significantly reduces the spurious wave content for most wave cases. Alternatively, figure 9(b) defines the spurious wave amplitude based on second-order force control, $A_{2}^{(22)}$, as a ratio of the corresponding value arising from first-order force control, $A_{1}^{(22)}$. In this latter case, ratios less than unity define the relative merit of second-order force control.

According to the theory outlined in part I, the amplitude of the spurious second-order wave should reduce to zero with the adoption of a second-order command signal. However, in practice the experimental error associated with the measurement of the water surface elevation becomes similar in magnitude to the spurious wave component, particularly as the wave frequency increases. For example, in wave case $f=48 / 64 \mathrm{~Hz}$ with a steepness of $H k / 2=0.16$ (table 1), the $10 \%$ dimensionless spurious wave ratio (figure 9a) corresponds to $0.8 \mathrm{~mm}$ of spurious wave amplitude. This is not substantially larger than the absolute experimental error of $\pm 0.5 \mathrm{~mm}$ associated with the wave gauges.

It has already been noted in relation to the first-order force control experiments that the results are less satisfactory for higher frequencies. The results presented in figure 9 suggest that this is equally true for second-order force control. In addition to the arguments outlined in $\S 4$, the second-order correction may also be affected by the fact that the systems drive mechanics are not considered in the theoretical model; this includes the motor with mechanical inertia and electrical inductance, the gearing system with inertia and the power amplifier. These components will add a small phase shift to the compensation signal and this may 

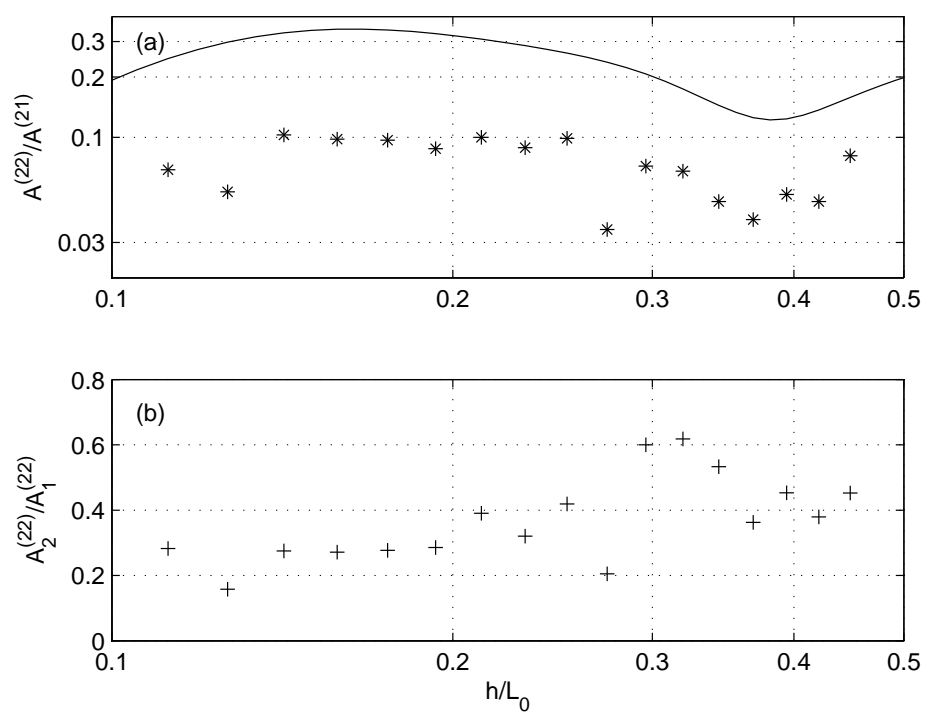

Figure 9: (a) Dimensionless, second-order, spurious wave content using second-order force control; - First-order force-control theory (for reference); * Second-order force-control experiment; (b) Ratio of spurious wave in second- and first-order force control experiment, $+A_{2}^{(22)} / A_{1}^{22}$.

become larger at higher frequencies. These effects will be considered in future work, but given the absolute magnitude of the second-order spurious wave in the higher frequency range they where not considered to be of primary importance to the present study.

Figures 10-13 show the wave profiles (or close-ups thereof) of two waves cases in which second-order force control has been applied successfully. Figure 10 concerns a wave frequency of $f=34 / 64 \mathrm{~Hz}$, with a steepness of $H k / 2=0.11$, and compares the wave profile arising from (a) first-order force control and (b) second-order force control with the Stokes second-order solution. Although the data relating to first-order force control (figure 10a) are in reasonable agreement with the Stokes solution (comparable to figure 5b), some important departures remain. Whilst these are not entirely eliminated by the adoption of second-order force control, they are substantially reduced (figure 10b); evidence of this being provided by the close-ups of the data records at locations (1)-(4) given on figure 11. Figures 12 and 13 provide a similar sequence of plots relating to a wave frequency of $f=48 / 64 H z$ and a steepness of $H k / 2=0.16$. Once again, these results demonstrate that there is a small but noticeable improvement in the wave quality following the adoption of second-order force control. 

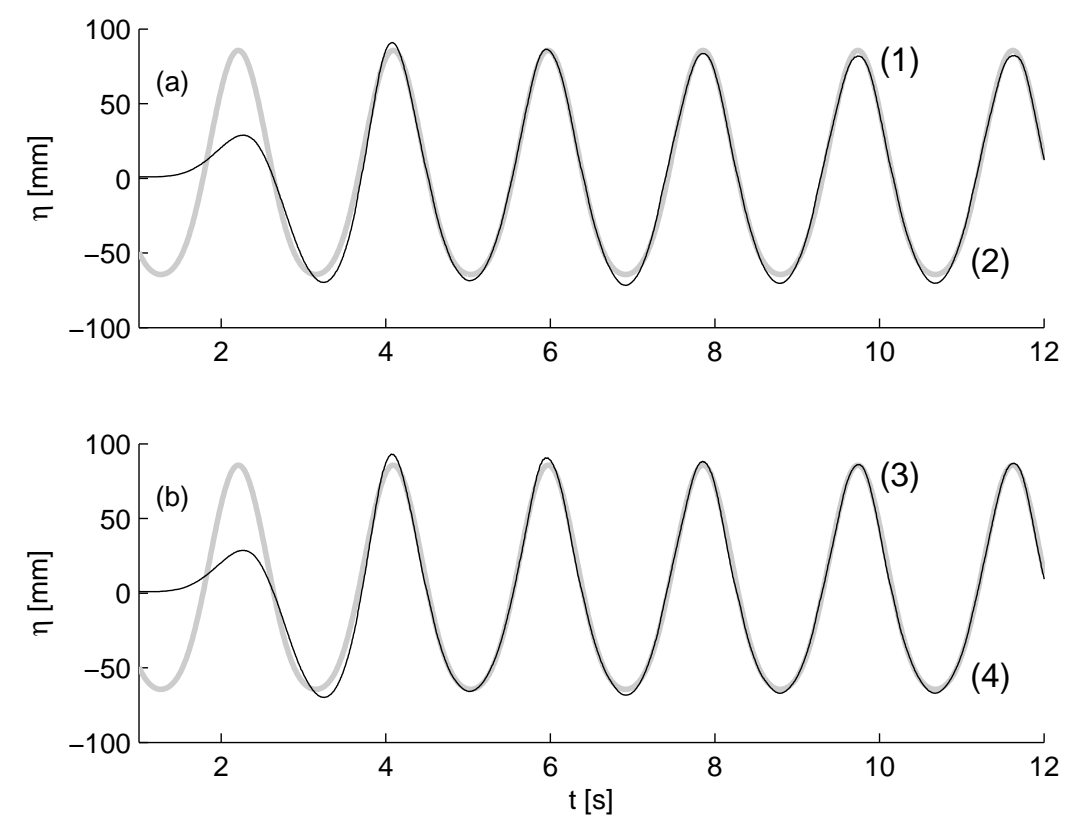

Figure 10: Surface elevation for wave case $f=34 / 64 H z, H k / 2=0.11$; (a) First-order force control, (b) Second-order force control, — Experiment, - Second-order Stokes solution; Note: the numbers (1) to (4) refer to locations that are considered in detail in figure 11
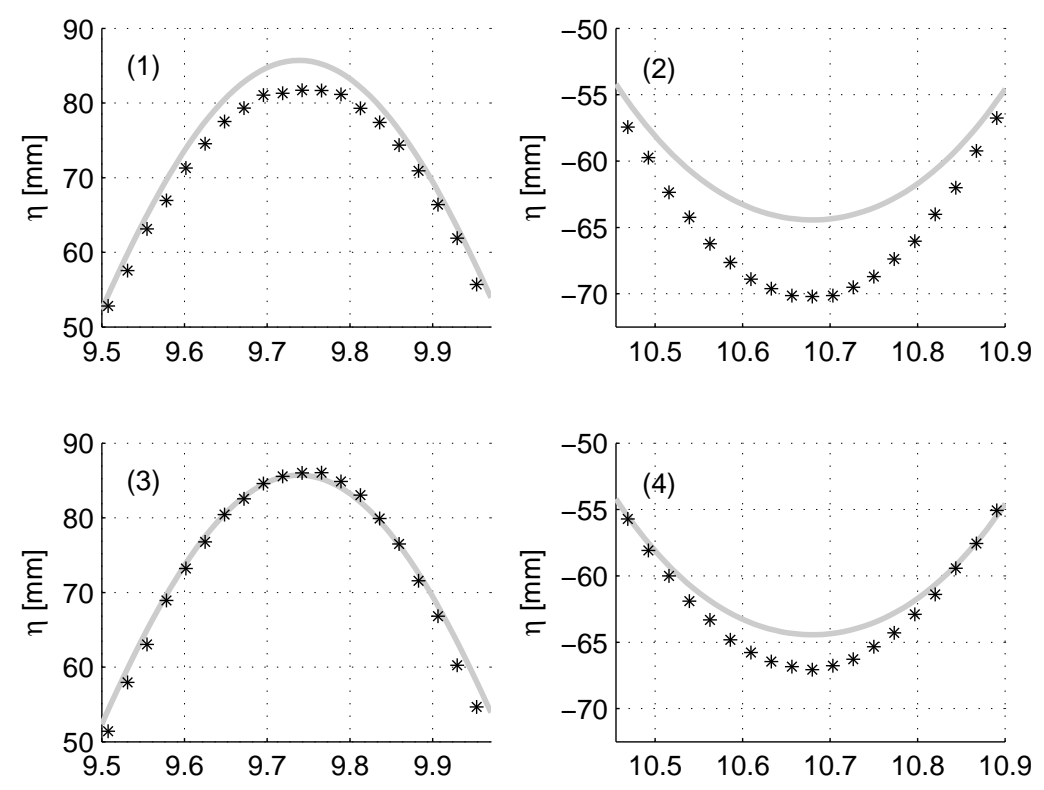

Figure 11: Close-up of the water surface elevation at locations (1) to (4) for wave case $f=34 / 64 \mathrm{~Hz}$, $H k / 2=0.11$ as labelled in figure $10 ; *$ Experiment, $\longrightarrow$ Second-order Stokes solution 

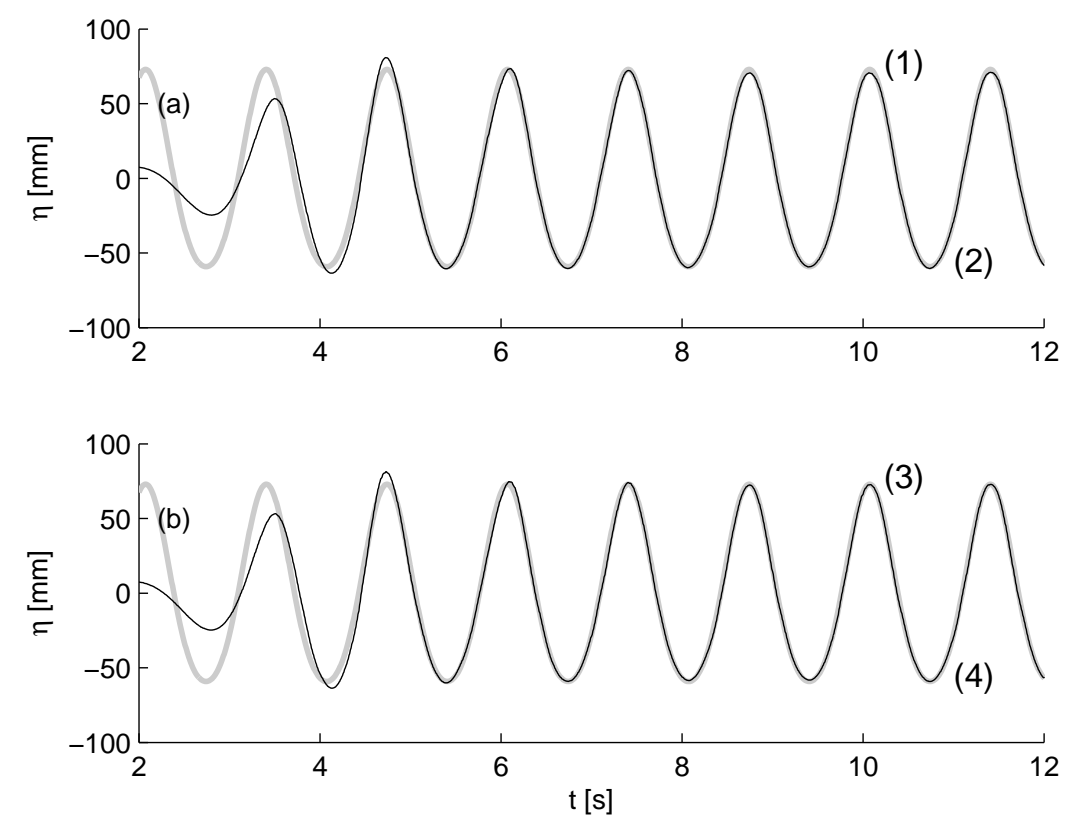

Figure 12: Surface elevation for wave case $f=48 / 64 H z, H k / 2=0.16$; (a) First-order force control, (b) Second-order force control, — Experiment, — Second-order Stokes solution; Note: the numbers (1) to (4) refer to locations that are considered in detail in figure 13
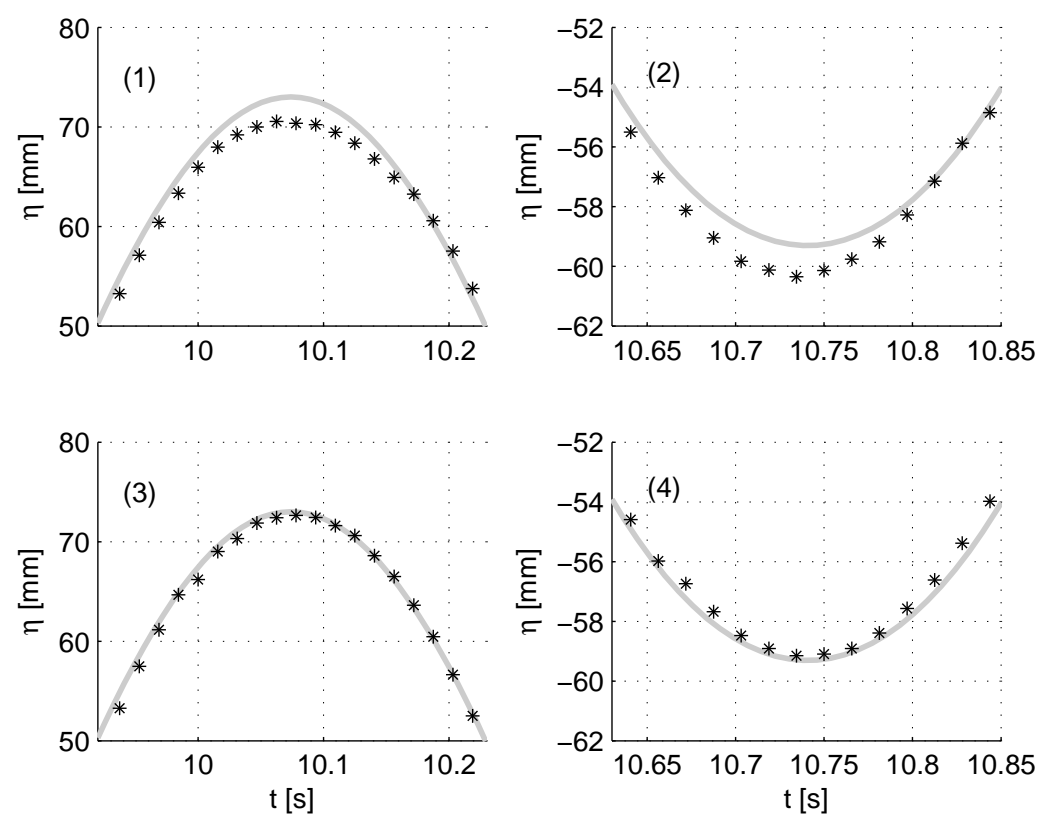

Figure 13: Close-up of the water surface elevation at locations (1) to (4) for wave case $f=48 / 64 \mathrm{~Hz}$, $H k / 2=0.16$ as labelled in figure $12 ; *$ Experiment, Second-order Stokes solution 


\section{Conclusions}

The force-feedback theory developed in part I has been compared to experimental observations and three key features of the theory verified. First, active absorption based upon first-order impedance matching has been shown to work well over a broad frequency band, and especially for low frequencies were traditional passive absorbers perform poorly. Second, a bottom-hinged flap-type wave maker driven by first-order force control produces significantly smaller second-order spurious waves when compared to an identical wave maker driven by first-order position control. This highlights the inherent advantages of a system driven with force-feedback control. Third, the validity of a second-order correction signal has been proven, leading to a further significant reduction in the spurious wave content. However, in respect of the latter, some deviations from the theory remain for higher frequency waves and the model may need to be expanded to include the drive system's dynamics. To date, these results are limited to flap-type wave makers generating regular waves. On-going work is considering random or irregular waves and seeking to expand the analysis to address piston-type wave machines.

\section{Acknowledgement}

The authors gratefully acknowledge Edinburgh Designs Ltd. for providing full access to their software and control systems.

\section{References}

Baldock, T. E., Swan, C. \& Taylor, P. H. (1996), 'A laboratory study of nonlinear surface waves on water.', Philosophical Transactions of the Royal Society of London A 354, 649-676.

Falnes, J. (2002), Ocean Waves and Oscillating Systems, Cambridge University Press.

Isaacson, M. (1991), 'Measurement of regular wave reflection', Journal of Waterway, Port, Coastal and Ocean Engineering 117(6), 553 - 569.

Johannessen, T. B. \& Swan, C. (2001), 'A laboratory study of the focusing of transient and directionally spread surface water waves.', Proc. Roy. Soc. Lond. A 457, 1-36.

Johannessen, T. B. \& Swan, C. (2003), 'On the nonlinear dynamics of focused wave groups in two and three dimensions.', Proc. Roy. Soc. Lond. A 459, 1021-1052.

Masterton, S. \& Swan, C. (2008), 'On the accurate and efficient calibration of a 3d wave basin', Ocean Engineering 35(8-9), 763 - 773.

Schäffer, H. A. (1996), 'Second-order wavemaker theory for irregular waves', Ocean Engineering 23, 47-88.

Sheikh, R. \& Swan, C. (2005), 'The interaction between steep waves and a vertical, surface-piercing column', Transactions of the ASME. Journal of Offshore Mechanics and Arctic Engineering 127(1), 31 - 8 .

Spinneken, J. \& Swan, C. (2008a), 'Second-order wave maker theory using force-feedback control. Part I. A new theory for regular wave generation', Ocean Engineering; Submitted for revision; .

Swan, C., Taylor, P. \& van Langen, H. (1997), 'Observations of wave-structure interaction for a multi-legged concrete platform', Applied Ocean Research 19(5-6), 309 - 327. 